\title{
ADDENDUM
}

\section{Self-assembly of self-limiting monodisperse supraparticles from polydisperse nanoparticles}

Yunsheng Xia, Trung Dac Nguyen, Ming Yang, Byeongdu Lee, Aaron Santos, Paul Podsiadlo, Zhiyong Tang, Sharon C. Glotzer and Nicholas A. Kotov

Nature Nanotechnology 6, 580-587 (2011); published online 21 August 2011; corrected online 26 August 2011; corrected after print 25 May 2012.

In this Article we should have mentioned previous works on the competition between van der Waals forces and/or other attractive shortrange forces and electrostatic long-range interactions in the assembly of different types of aggregates. To our knowledge this issue was first studied by Zeleny in 1914 in experiments on the instability of charged droplets, with the competing forces being electrical repulsion and surface tension ${ }^{1,2}$, and it was later discussed by Taylor in $1964^{3}$. The idea that negatively charged nanoparticles can agglomerate to form larger particles via magic-number progression was proposed in ref. 4 in 1984. In 1985 Ramsden reported that CdS nanoparticles formed aggregates of limited dimensions ${ }^{5}$, and in 1987 he explained the stability of polydisperse superspheres containing a few tens of nanoscale particles in terms of the balance between long-range electrostatic repulsion and short-range attractive dispersion forces ${ }^{6}$. In the early 1990s Zukoski and co-workers also explored how equilibrium between long- and short-range forces influenced the size of nanoparticle clusters ${ }^{7,8}$.

The clear difference between our work and that reported in refs 1-6 is that the supraparticles (superspheres) in our experiments are much more uniform in size and shape. This has allowed us to develop a new phenomenological model of supraparticle formation that, in turn, has led to the observation of a number of nanoparticle structures that had not been observed before, including colloidal crystals made of supraparticles and various core-shell systems (including some that resemble viruses). The main difference between our work and that reported in refs 7,8 is that the clusters observed in those experiments were transient in nature and their self-limiting properties were less obvious. We also demonstrate the generality of the phenomenon by making supraparticles from combinations of two materials.

\section{References}

1. Zeleny, J. The electrical discharge from liquid points and a hydrostatic method of measuring the electric intensity at their surfaces. Phys. Rev. 3, 69-91 (1914).

2. Zeleny, J. On the conditions of instability of electrified drops with applications to the electrical discharge from liquid points. Proc. Camb. Phil. Soc. 18, 71-83 (1915).

3. Taylor, G. I. Disintegration of water drops in an electric field. Proc. R. Soc. Lond. A 280, 383-397 (1964).

4. Fojtik, A., Weller, H., Koch, U. \& Henglein, A. Photo-chemistry of colloidal metal sulfides 8. Photo-physics of extremely small CdS particles: Q-state CdS and magic agglomeration numbers. Ber. Bunsenges. Phys. Chem. 88, 969-977 (1984).

5. Ramsden, J. J. The nucleation and growth of small CdS aggregates by chemical reaction. Surf. Sci. 156, 1027-1039 (1985).

6. Ramsden, J. J. The stability of superspheres. Proc. R. Soc. Lond. A 413, 407-414 (1987).

7. Biggs, S., Chow, M. K. \& Zukoski, C. F. The role of colloidal stability in the formation of gold sols. J. Colloid Interface Sci. 160, 511-513 (1991).

8. Look, J. L. \& Zukoski, C. F. Alkoxide-derived titania particles: use of electrolytes to control size and agglomeration levels. J. Am. Ceram. Soc. 75, 1587-1595 (1992). 